\title{
Experience of Fully Awake Craniotomy for Supratentorial Lesions: A Single Institution Study
}

\author{
Erdal COSKUN ${ }^{1}$, Fatih YAKAR ${ }^{1}$, Serkan CIVLAN ${ }^{1}$, Eyup BAYKARA², Batuhan BAKIRARAR ${ }^{3}$, Emrah EGEMEN ${ }^{1}$ \\ ${ }^{1}$ Pamukkale University School of Medicine, Department of Neurosurgery, Denizli, Turkey \\ ${ }^{2}$ Bahcelievler Medicana International Hospital, Istanbul, Turkey \\ ${ }^{3}$ Ankara University School of Medicine, Department of Biostatistics, Ankara, Turkey
}

Corresponding author: Fatih YAKAR yakarneurosurgery@gmail.com

\section{ABSTRACT}

AIM: To report and to discuss our experience of awake craniotomy for the treatment of supratentorial lesions.

MATERIAL and METHODS: We included patients who underwent awake craniotomy for supratentorial tumors between 2007 and 2018. A bipolar stimulation probe was used for patients with eloquent area lesions. The demographic features, presenting symptoms, comorbidities, localization, histopathology, pre- and postoperative Karnofsky performance status, mean operation length, mean length of hospital stay, and intraoperative and postoperative complications were recorded.

RESULTS: We included 250 patients (age, $53.5 \pm 15.3$ years; range, 15-90 years; 105 females and 145 males) mostly with metastasis (46\%). The tumor resection rate was $90 \pm 3.6 \%$. Of 30 patients (12\%) who experienced an increase in weakness, 26 experienced improvements within three days, and the remainder had permanent symptoms. Intraoperative and postoperative seizures occurred in three (1.2\%) and seven (2.8\%) patients, respectively, which were controlled by antiepileptic drugs. Dysphasia occurred intraoperatively in seven patients $(2.8 \%)$ but improved in a month. The mean follow-up duration was $31.8 \pm 11.9$ months (range, 7-70 months). No mortality was seen during hospitalization.

CONCLUSION: Awake procedures are a good option in supratentorial lesions to avoid the complications of general anesthesia for patients in poor medical condition. To obtain maximal tumor resection and to maintain better quality of life, neurosurgeons should opt for awake craniotomy when necessary.

KEYWORDS: Awake craniotomy, Metastasis, Karnofsky performance status, Quality of life

ABBREVIATIONS: AC: Awake craniotomy, iUSG: Intraoperative ultrasonography, CUSA: Cavitron Ultrasound Surgical Aspirator, CT: Computed tomography, KPS: Karnofsky performance status, MRI: Magnetic resonance imaging, LGG: Low-grade glioma

\section{INTRODUCTION}

I n 1886, Sir Victor Horsley performed the first awake craniotomy (AC) to find the epileptic focus (30). However, earliest evidence comes from archeological data (8). Of 214 skulls unearthed in Peru, trephination was successfully performed with a healing rate of $55 \%$. Given that these were performed before the era of general anesthesia, coca leaves may have been used as local anesthetics (8).
Modern surgical principles of $A C$ that rely on local anesthesia with intermittent sedation and analgesia were published by Wilder Penfield and André Pasquet 60 years ago (10). Since then, $\mathrm{AC}$ techniques have been developed in collaboration between anesthesiologists and neurosurgeons for the treatment of epilepsy and tumors in eloquent areas (36). Although there is a perception that AC should be limited to lesions in eloquent areas, it can be used to avoid complications
Erdal COSKUN : D : 0000-0002-2816-0722

Fatih YAKAR (D): 0000-0001-7414-3766

Serkan CIVLAN (D) : 0000-0001-8915-8186 $\begin{array}{ll}\text { Eyup BAYKARA } & \text { (1) : 0000-0001-9984-0322 } \\ \text { Batuhan BAKIRARAR (1) : 0000-0002-5662-8193 } \\ \text { Emrah EGEMEN } & \text { (D) : 0000-0003-4930-4577 }\end{array}$ 
of general anesthesia when treating supratentorial lesions $(36,37)$. Furthermore, the instant intraoperative feedback available in $A C$ is invaluable in helping neurosurgeons decide when to terminate the procedure.

At our institute, we use AC as a standard surgical procedure to improve the quality of life of patients with supratentorial metastases. In this study, we present use of AC for patients with supratentorial metastases and other lesions.

\section{MATERIAL and METHODS}

\section{Study Design and Patient Population}

We conducted a retrospective single-center study of patients with supratentorial lesions treated with AC between 2007 and 2018. Although awake surgery is recommended for supratentorial lesions, it is presented as the first choice for patients with comorbidities and a Karnofsky performance status (KPS) score $<100$ points. Written informed consent was obtained from the patients before enrolment and the procedure was explained to the patients using videos of operations. A preoperative agreement was reached with the patient on whether the operation would continue if seizures, weakness, or speech disorders developed. We excluded patients with severe aphasia or cognitive disorders, depression, psychotic disorders, and claustrophobia.

\section{Anesthesia Care}

All patients were involved in an awake-awake-awake procedure and received premedication (e.g., a benzodiazepine), venous or arterial lines were placed in the forearm, and a urinary catheter was inserted. Heart rate, blood pressure, and pulse rate (using a pulse oximetre) were monitored for all cases.

The surgical field was blocked with $0.75 \%$ ropivacaine (Ropivacain $^{\circledR}$, Fresenius Kabi, Bad Homburg, Germany) and 1:200000 epinephrine (3). Remifentanil $\left(20 \mu \mathrm{g} / \mathrm{mL}\right.$; Ultiva ${ }^{\circledR}$, Glaxo Smith Kline, Hamburg, Germany) was available for continuous infusion if required. An antibiotic ( $2 \mathrm{~g}$ cefazolin), an anticonvulsant (500 mg levetiracetam or $250 \mathrm{mg}$ phenytoin) for patients with a history of seizure, and a corticosteroids (8 mg dexamethasone) were administered to all patients before the skin incision.

\section{Operative Technique}

Operations were performed in supine or lateral positions, according to the tumor location, without using skull clamps. A disposable surgical skin stapler was used to predict the tumor location, and CT images were obtained with the surgical staple before surgery as a low-cost and feasible navigation system. This method was preferred in the early stages because of the absence of a neuronavigation system in our clinic. However, since the system provided later required skull clamp use, the $\mathrm{CT}$, and surgical staple method was habituated in this group of patients. Pereira et al. also did not use a skull clamp and the neuronavigational system in patients with tumors in the eloquent area (29). Intraoperative ultrasonography (iUSG; Elegra; Siemens, Erlangen, Germany) and the Cavitron Ultrasound Surgical Aspirator (CUSA) were set up before standard craniotomy was performed.
A bipolar stimulation probe (Avalanche ${ }^{\circledR}$ XT, Dr. Langer Medical $\mathrm{GmbH}$, Waldkirch, Germany) was used for patients with eloquent area lesions. For cortical stimulation, $50 \mathrm{~Hz}$ $0.5 \mathrm{~ms}$ biphasic square waves were used, starting with $1 \mathrm{~mA}$ stimulus and increasing in steps of $0.5 \mathrm{~mA}$ to a maximum of $10 \mathrm{~mA}$ stimulus. The choice of intraoperative neurological evaluation depended on the anatomic location of the surgery. Typically, we assessed object-naming or verb-generation exercises, counting, reading, visual-field monitoring, and mathematics.

\section{Postoperative Care}

We performed routine cranial CT after-surgery and transferred the patients to the postoperative care unit to be observed for 2 hours. Patients without neurological or hemodynamic problems were taken to the ward after a joint decision by the neurosurgeon and anesthesiologist.

\section{Data Collection}

The following data were recorded for patients: age, gender, preoperative, and postoperative KPS score, pathology, comorbidity, intraoperative parameter, clinical result, and complication. We obtained patient information from the clinical computer registry system, by chart review, and by in-person or telephone-based interviews.

\section{Statistical Analysis}

Data analysis was performed using SPSS 11.5 Windows version. Mean \pm standard deviation and median (minimummaximum) for quantitative variables and number (\%) for qualitative variables are given as described. When the difference between the two dependent quantitative measurement values was statistically significant, the Wilcoxon signed-rank test was used because normality assumptions were not met. The statistical significance level was set at 0.05 .

\section{RESULTS}

\section{Demography of Patients}

We performed AC on 250 patients (105 females and 145 males) with supratentorial tumors. The clinical and operative details are reported in Tables I, II. The mean age was $53.5 \pm$ 15.3 years (range: $15-90$ years). The most common symptom presented was weakness. Patients also presented with seizure, headache, eloquence deficits (e.g., sensory, visual, cognitive, or speech), and scalp lumps. The most common comorbidity was chronic obstructive pulmonary disease (17.6\%), followed by hypertension (12\%), pneumonectomy $(12 \%)$, diabetes mellitus $(11.2 \%)$, obesity $(8 \%)$, coronary artery diseases $(5.6 \%)$, dialysis $(2.4 \%)$, pulmonary embolism $(0.8 \%)$, hypothyroidism $(2 \%)$, heart valve replacement with warfarin use $(0.8 \%)$, and nephrectomy $(0.8 \%)$.

\section{Characteristics of Lesions}

More than half the patients had right-sided tumors $(54.4 \%$, $\mathrm{n}=136)$ and involved the parietal lobe (38.8\%), frontal $(29.2 \%)$, temporal (16.4\%), and occipital (8.4\%) lobes and the insula $(6 \%)$ and thalamus (1.2\%) (Table II). The most common tumor 
Table I: The Demographic Features, Symptoms, Pre/Postoperative Karnofsky Performance Status, and Comorbidities of the Patients at the Time of Admission

\begin{tabular}{|c|c|c|c|}
\hline Characteristic & Value & & \\
\hline Number of the patients & 250 & & \\
\hline Age, Mean (min-max ) (years) & $53.5 \pm 15.3(15-90)$ & & \\
\hline \multirow[t]{5}{*}{$\operatorname{Sex}(F, M)$} & $105 \mathrm{~F}, 145 \mathrm{M}$ & & \\
\hline & & $\mathbf{n}$ & $\%$ \\
\hline & Weakness & 97 & \\
\hline & Seizure & 49 & \\
\hline & Headache & 35 & \\
\hline \multirow{10}{*}{ Symptoms } & Sensory deficit & 30 & \\
\hline & Visual deficit & 27 & \\
\hline & Cognitive deficit & 25 & \\
\hline & Speech deficit & 13 & \\
\hline & Scalp lump & 2 & \\
\hline & COPD & 44 & 17.6 \\
\hline & $\mathrm{HT}$ & 30 & 12 \\
\hline & Pneumonectomy & 30 & 12 \\
\hline & $\mathrm{DM}$ & 28 & 11.2 \\
\hline & Obesity & 20 & 8 \\
\hline \multirow[t]{6}{*}{ Comorbidities } & CAD & 14 & 5.6 \\
\hline & Dialysis & 6 & 2.4 \\
\hline & Hypothyroidism & 5 & 2 \\
\hline & Pulmonary Embolism & 2 & 0.8 \\
\hline & HVR (warfarin sodium use) & 2 & 0.8 \\
\hline & Nephrectomy & 2 & 0.8 \\
\hline
\end{tabular}

\section{Pre/postoperative KPS score, Mean $83.7 \pm 10.4$ / $88.2 \pm 8.9$}

F: Female, M: Male, COPD: Chronic obtructive pulmonery disease, HT: Hypertension, DM: Diabetes mellitus, CAD: Coroner artery disease, HVR: Heart valve replacement, KPS: Karnofsky performance status point.

Table II: The Summary of Localization and Histopathological Features of Lesions

\begin{tabular}{|c|c|c|c|c|}
\hline & & $\mathbf{n}$ & $\mathrm{R} / \mathrm{L}$ & $\%$ \\
\hline \multirow{5}{*}{ Localization } & Parietal & 97 & $53 / 44$ & 38.8 \\
\hline & Frontal & 73 & $39 / 34$ & 29.2 \\
\hline & Temporal & 41 & $20 / 21$ & 16.4 \\
\hline & Insula & 15 & $11 / 4$ & 6 \\
\hline & Thalamus & 3 & $1 / 2$ & 1.2 \\
\hline & Glioblastoma, NOS & 61 & & 24.4 \\
\hline & Anaplastic astrocytoma, NOS & 23 & & 9.2 \\
\hline & Oligodendroglioma, NOS & 17 & & 6.8 \\
\hline & Meningioma & 12 & & 4.8 \\
\hline & Falx & 7 & & 2.8 \\
\hline & Parasagittal & 4 & & 1.6 \\
\hline & Radiation Necrosis & 2 & & 0.8 \\
\hline & Arteriovenous Malformation & 2 & & 0.8 \\
\hline & Aneurysm & 1 & & 0.4 \\
\hline & Hydatid Cyst & 1 & & 0.4 \\
\hline & Diffuse large B-cell lymphoma of the CNS & 1 & & 0.4 \\
\hline & Arachnoid Cyst & 1 & & 0.4 \\
\hline & Solitary Fibrous tumor/Haemangiopericytoma & 1 & & 0.4 \\
\hline
\end{tabular}

R/L: Right/Left, NOS: Not otherwise specified, CNS: Central nervous system. 
types were metastasis (46\%) and glioblastoma not otherwise specified (24.4\%) (Figure 1A-F).

\section{Intraoperative Findings}

Intraoperative seizures occurred in three patients (1.2\%). One patient had a history of drug abuse. All patients had a small degree of brain swelling. However, the operations were performed after administering remifentanil and additional

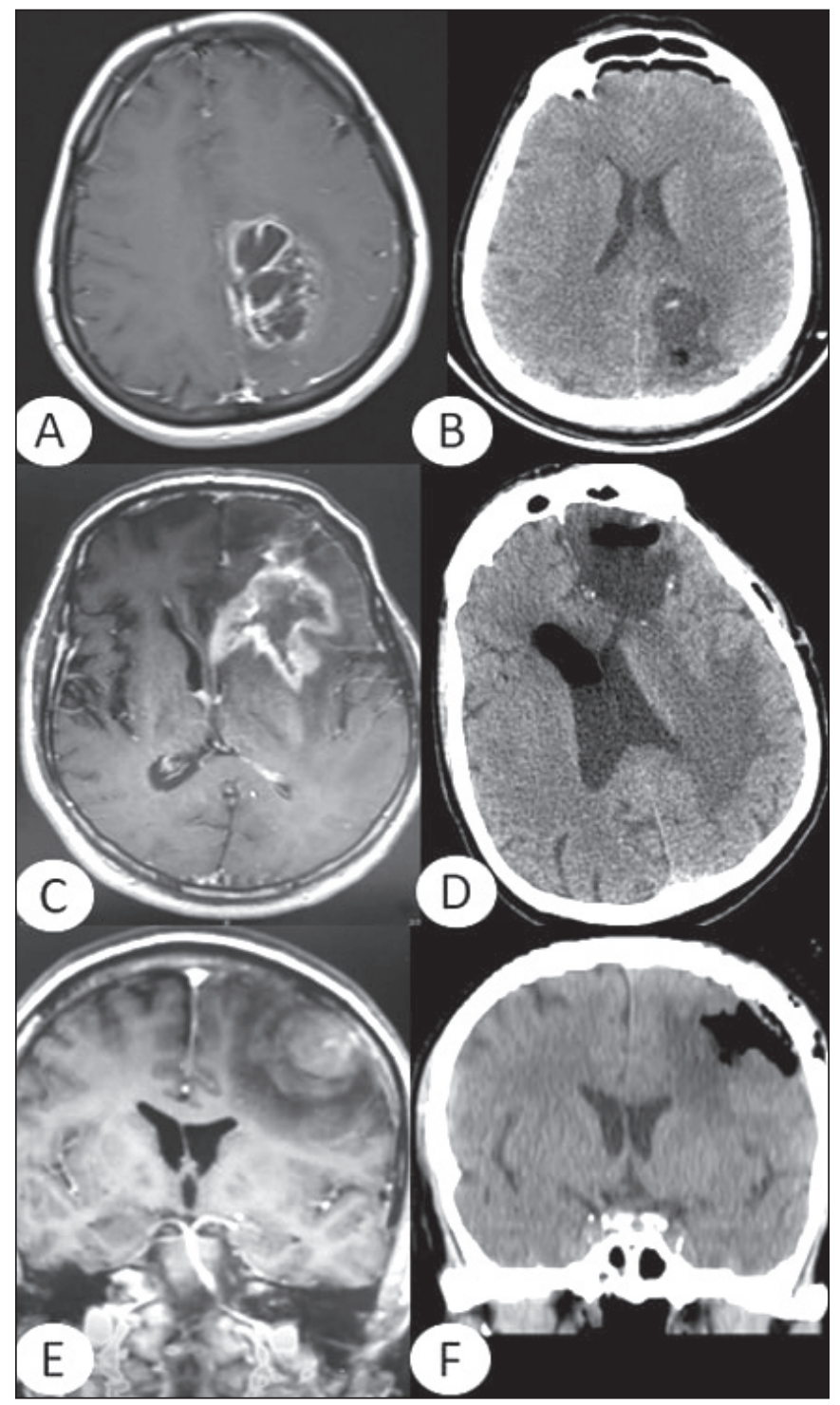

Figure 1A-F: Imaging results of three representative cases.

A, B) A 25-year-old female admitted with recurrent anaplastic astrocytoma. T1-weighted magnetic resonance imagination (MRI) showed a large cystic hyperintense lesion. C, D) A 65-year-old male who underwent craniotomy for glioblastoma was admitted to our hospital with right hemiparesis. Contrast-enhanced T1-weighted MRI showed a recurrent lesion. A histopathological examination verified the previous diagnosis. E, F) A 54-year-old male with a previously diagnosed as malign melanoma was admitted to our hospital with dizziness and hemiparesis. MRI revealed a metastatic lesion on contrast-enhanced T1-weighted MRI. A histopathological examination verified the diagnosis of metastasis. doses of antiepileptic drugs. No seizures occurred following intraoperative cortical stimulation. The median operation time was 90 minutes $(84.5 \pm 23.5)$ and the mean length of hospital stay was $5(4.9 \pm 1.6)$ days. CUSA and iUSG were used in $22.8 \%$ and $26 \%$ patients, respectively. Gross total resection $(90 \pm 3.6 \%)$ was achieved during surgery.

\section{Neurological Complications}

The overall (intraoperative and postoperative) neurological complication rate was $18.8 \%(n=47)$, with the effects being permanent in $1.6 \%(n=4)$. Dysphasia occurred intraoperatively in seven patients (2.8\%), thus requiring termination of the operations; however, it improved within a month. Intraoperative arm contraction in three patients (1.2\%) also required termination of the operation. Thirty (12\%) patients experienced weakness in the postoperative period, which improved within three days for 26 patients; however, four patients experienced permanent weakness.

There was a significant increase in the mean KPS score from $83.7 \pm 10.4$ preoperatively to $88.2 \pm 8.9$ postoperatively $(p<$ 0.001 ). The mean follow-up duration was $31.8 \pm 11.9$ months (range, 7-70).

Tonic-clonic seizures occurred in seven patients $(2.8 \%)$ in the first postoperative day. After airway safety was ensured, cranial CT images were obtained to exclude hematoma or edema. No pathology was detected on the CT scan. In all cases, seizures were controlled with antiepileptic drugs and did not recur. A postoperative hematoma was seen on the CT immediately after the operation in one patient $(0.4 \%)$, who underwent urgent re-craniotomy. Wound healing problems occurred in 15 patients $(6 \%)$, of which three required further operations to treat cerebrospinal fluid leakage. No mortality was seen during hospitalization.

\section{DISCUSSION}

The development of effective locoregional anesthesia is a milestone in the use of $\mathrm{AC}$ as a standard operative approach (9). During the operation, additional analgesia is not needed; local injection of the anesthetics to the surgical field is often sufficient (11). Even under general anesthesia and opioid administration, skull clamp placement can increase heart rate and blood pressure by $15 \%$ and $43 \%$, respectively (22). Locoregional anesthesia reduces this autonomic response and provides a stable hemodynamic course (5). The main goal is to ensure patient comfort while facilitating accurate cortical mapping (28). However, the AC approach offers many benefits over classical methods, including having fewer iatrogenic injuries, the ability to avoid general anesthesia and invasive monitoring, shorter intensive care and hospital stays, and lower expense burden $(1,4,17,19,26,37)$.

AC requires administration of local anesthesia (31). The injection sites can range from a specific region in the scalp (or nerve) to a dispersed surgical field (16). Anesthetic care is performed in one of three ways for AC. First, the sleep-awakesleep technique involves anesthesia while fixing the head with a skull clamp to perform a craniotomy, but requires that 
consciousness be regained for cortical mapping. The second choice involves mild sedation to control anxiety and pain while maintaining careful monitoring (31). Craniotomy and closure do not require patient cooperation; therefore, deep sedation can be used in these stages (11). Finally, excluding sedation and relying on analgesia alone is the fully awake option (i.e., the awake-awake-awake procedure). Intracranial pressure due to hematoma or brain edema or straining during emergence may lead to neurological worsening, which is imperceptible until the patient becomes fully awake. In contrast, fully awake procedures allow us to know the neurological state instantaneously, potentially allowing us immediate management at the time of worsening of condiditon.

For a successful procedure, one of the most critical factors is appropriate patient selection (35). There are no universally accepted criteria for patient selection. However, some institutions have reported specific contraindications such as a patient with reluctance, claustrophobia, anxiety disorders, confusion/somnolence, apparent dysphagia, alcohol, or drug dependence, chronic pain disorders, restless leg syndrome, low pain tolerance, morbid obesity, obstructive sleep apnea, and uncontrolled cough (38). The procedure is not recommended for meningiomas near vascular sinuses and in cases where the amount of bleeding is expected to exceed 750-1000 $\mathrm{mL}$ (38), nor is it recommended for occipital lobe tumors and in patients younger than 12 years of age (3). In 1954, Pasquet defined the age limit as ten (27). Although the procedure is not recommended for patients under ten years of age (3), it can be performed at clinics with more experience (21). To date, the youngest patient to have undergone AC was nine years old (21), while the youngest patient in our series was 15 years old. Grossman et al. reported that AC was a safe and well-tolerated procedure for patients older than 65 years of age (15). However, high intracranial pressure should not be considered an obstacle to $A C$ because brain relaxation is induced during the procedure (37). A well-known cause of unsuccessful sedation is hypertension (35), though AC can also be limited by insufficient pain control, airway obstruction, emesis, and intraoperative seizures (10). Contrary to reports in the literature, we do not consider tumor size or location to be contraindications at our institute (14).

In this study, AC was used to treat lesions in non-eloquent areas to compensate for the health status and comorbidities typical of patients with cranial metastasis. The primary tumor site (mostly the lung) results in high morbidity under general anesthesia. Therefore, we anticipated that awake procedures may reduce anesthesia-related morbidity. Indeed, Grossman et al. performed AC among 90 elderly patients and concluded that aggressive tumor resection by AC might improve survival, even in patients with multisystem issues (15). Quality of life, measured with the mean KPS score (41), increased postoperatively in our cohort, indicating improvement of quality of life.

Prolonged survival and decreased rate of malignant transformation are possible with aggressive and early surgery for low-grade gliomas (LGG). Maximum tumor resection leads to improved seizure control and avoids the development of permanent neurological deficit (40). Duffau stated that for patients with LGG with no or few complaints at diagnosis, no options other than AC are available (12). Awake procedures also allow neurosurgeons to evaluate the structural and functional integrity by cortical mapping during tumor resection (28). Radiologic evaluation and intraoperative motor and speech mapping during $A C$ are essential tools for achieving good neurological outcomes with a lower rate of permanent deficits $(13,20,40)$.

AC was used as a standard surgical approach for supratentorial tumors in a prospective trial of 200 consecutive patients (37), where brain mapping and new postoperative neurological deficit rates were $97.5 \%$ and $13 \%$ (4.5\% permanent), respectively, while the intraoperative seizure rate was $5.6 \%$ (one patient required general anesthesia). In another study, AC was routinely and non-selectively performed for supratentorial tumors in 610 patients (36). Brain mapping was performed in 511 of the patients intraoperatively, revealing that 115 and 396 patients had tumors in the eloquent and non-eloquent areas, respectively. Neurological worsening occurred during brain mapping in 78 patients (15.3\%), and 25 patients suffered from intraoperative seizures (two required general anesthesia). Boetto et al. reviewed 374 patients who underwent $A C$ and reported an intraoperative seizure rate of $3.4 \%$, with all cases resolved by irrigation with cold Ringer's lactate (6). In contrast, Keles et al. reported that new postoperative neurological deficits developed in $20 \%$ patients despite the use of intraoperative subcortical stimulation mapping for gliomas under general anesthesia (18). Intraoperative seizures, arguably the most crucial reason for terminating awake procedures, occurred in three patients (1.2\%) in our series.

Even though intraoperative mapping and neuromonitoring do not always show sudden neurological changes during surgery for LGG, the examination of a patient's neurological status can provide instant data. Given that mapping prolongs the length of the operation, we chose not to use cortical mapping or neuromonitoring. Furthermore, negative findings allow for safe surgical resection, while stimulation close to the eloquent areas may increase new postoperative deficits (20). Notably, the permanent neurological worsening rate in our series was $1.6 \%$, which is quite low compared with that of other series using cortical mapping. In our opinion, neurosurgeons should rely on neurological examination more than on mapping or neuromonitoring.

Skin incision, muscle and periosteal dissection, craniotomy, and dural incision are painful procedures. Touching a big vessel during tumor resection also causes severe pain (1). In case pain occurs, the operation should be halted, remifentanillike sedatives should be administered, and continued only after patient cooperation (7). In our experience, nausea and vomiting also tend to present when the surgeon enters the ventricles, so an antiemetic should be used before such procedures.

Reports in the literature indicate that the use of iUSG increases the rate of excision $(25,33)$. The apparent benefits of iUSG are anatomical information support and functional monitoring (24), making it superior to intraoperative neuromonitoring in detecting residues of LGG and determining cortical incisions 
for subcortical lesions. Moreover, utilizing CUSA to highvolume tumors can help to shorten the operation time. Tumor size and accessibility do not limit the use of $A C$ when using CUSA.

The length of surgery was detected to be 132 minutes by Sacko et al. for AC with brain mapping (32). Taylor and Bernstein stated that operating room time was 195 minutes. Our median operation time was 90 minutes, and it is a quite short time contrary to the literature (37).

Additionally, the length of hospitalization appears to be consistently shorter with $\mathrm{AC}$ than with procedures performed under general anesthesia. Blanshard et al. reported a median hospital stay of 4 days for 241 patients who underwent nonselective AC, while Sanus et al. reported an average hospital stay of 5 days in their review of 25 patients $(4,34)$. Grossman et al. also reported similarly short hospital stays for elderly patients (15). A reason for this reduced length of stay is that the dysphoria following general anesthesia is typically not seen in these patients (37). The length of hospital stay in our series was $4.9 \pm 1.6$ days, which is similar to that reported in the literature.

According to Khu et al., a patient's understanding of the AC procedure reduces anxiety and leads to greater trust (19). Cooperation and confidence between the surgical team and patient is essential for the success of AC (2). Indeed, enhanced patient tolerance $(19,23,39)$, and lower complication rates increase the availability of $\mathrm{AC}$, irrespective of eloquent area involvement $(4,36,37)$.

\section{Limitations}

The study has some limitations. An accurate comparison with general anesthesia-related craniotomy cannot be made based on this study; this will require a randomized controlled study. Therefore, the actual effectiveness of the procedure cannot be determined with certainty.

\section{CONCLUSION}

Awake procedures can be performed routinely for all supratentorial tumors to permit maximum and safe resection without the complications of general anesthesia. Patient compliance is a prerequisite, and it is our experience that intraoperative neurological examination offers superior information to cortical mapping and neuromonitoring. $A C$ is not only feasible but also causes no additional morbidity or mortality.

\section{ACKNOWLEDGMENTS}

Turkish Neurosurgical Society partly supports preparation for publication of this article.

\section{REFERENCES}

1. Akay A, Islekel S: Awake craniotomy procedure: Its effects on neurological morbidity and recommendations. Turk Neurosurg 28(2):186-192, 2018
2. Bajunaid KM, Ajlan AM: Awake craniotomy. A patient's perspective. Neurosciences (Riyadh) 20(3):248-252, 2015

3. Berger MS: The impact of technical adjuncts in the surgical management of cerebral hemispheric low-grade gliomas of childhood. J Neurooncol 28:129-155, 1996

4. Blanshard HJ, Chung F, Manninen PH, Taylor MD, Bernstein M: Awake craniotomy for removal of intracranial tumor: Considerations for early discharge. Anesth Analg 92(1):89-94, 2001

5. Bloomfield EL, Schubert A, Secic M, Barnett G, Shutway F, Ebrahim ZY: The influence of scalp infiltration with bupivacaine on hemodynamics and postoperative pain in adult patients undergoing craniotomy. Anesth Analg 87(3):579-582, 1998

6. Boetto J, Bertram L, Moulinié G, Herbet G, Moritz-Gasser S, Duffau $\mathrm{H}$ : Low rate of intraoperative seizures during awake craniotomy in a prospective cohort with 374 supratentorial brain lesions: Electrocorticography is not mandatory. World Neurosurg 84(6):1838-1844, 2015

7. Bohyun S, Hee-Soo K, Jin-Woo P, Hyo-Jin B, Jin-Tae K, Chong Sung K: Anesthetic management with scalp nerve block and propofol/remifentanil infusion during awake craniotomy in an adolescent patient. Korean J Anesthesiol 59 Suppl:S179-S182, 2010

8. Bulsara KR, Johnson J, Villavicencio AT: Improvements in brain tumor surgery: The modern history of awake craniotomies. Neurosurg Focus 18(4):e5, 2005

9. Chaki T, Sugino S, Janicki PK, Ishioka Y, Hatakeyama $Y$, Hayase T, Kaneuchi-Yamashita M, Kohri N, Yamakage M: Efficacy and safety of a lidocaine and ropivacaine mixture for scalp nerve block and local infiltration anesthesia in patients undergoing awake craniotomy. J Neurosurg Anesthesiol 28(1):1-5, 2016

10. Costello TG, Cormack JR: Anaesthesia for awake craniotomy: A modern approach. J Clin Neurosci 11(1):16-19, 2004

11. Delion M, Terminassian A, Lehousse T, Aubin G, Malka J, N'Guyen S, Mercier P, Menei P: Specificities of awake craniotomy and brain mapping in children for resection of supratentorial tumors in the language area. World Neurosurg 84(6):1645-1652, 2015

12. Duffau $\mathrm{H}$ : Is non-awake surgery for supratentorial adult low-grade glioma treatment still feasible? Neurosurg Rev 41(1):133-139, 2018

13. Duffau H, Lopes M, Arthuis F, Bitar A, Sichez JP, Van Effenterre R, Capelle L: Contribution of intraoperative electrical stimulations in surgery of low grade gliomas: A comparative study between two series without $(1985$ - 96) and with (19962003) functional mapping in the same institution. J Neurol Neurosurg Psychiatry 76:845-851, 2005

14. Dziedzic T, Bernstein M: Awake craniotomy for brain tumor: Indications, technique and benefits. Expert Rev Neurother 14:1405-1415, 2014

15. Grossman R, Nossek E, Sitt R, Hayat D, Shahar T, Barzilai O, Gonen T, Korn A, Sela G, Ram Z: Outcome of elderly patients undergoing awake- craniotomy for tumor resection. Ann Surg Oncol 20(5):1722-1728, 2013 
16. Guilfoyle MR, Helmy A, Duane D, Hutchinson PJA: Regional scalp block for postcraniotomy analgesia. Anesth Analg 116 (5):1093-1102, 2013

17. Heifets BD, Crawford E, Jackson E, Brodt J, Jaffe RA, Burrridge MA: Case report of an awake craniotomy in a patient with Eisenmenger Syndrome. Anesth Analg 10(9):219222, 2018

18. Keles GE, Lundin DA, Lamborn KR, Chang EF, Ojemann G, Berger MS: Intraoperative subcortical stimulation mapping for hemispherical perirolandic gliomas located within or adjacent to the descending motor pathways: Evaluation of morbidity and assessment of functional outcome in 294 patients. $J$ Neurosurg 100(3):369-375, 2004

19. Khu KJ, Doglietto F, Radovanovic I, Taleb F, Mendelsohn D, Zadeh G, Bernstein M: Patients' perceptions of awake and outpatient craniotomy for brain tumor: A qualitative study. $J$ Neurosurg 112(5):1056-1060, 2010

20. Kim SS, McCutcheon IE, Suki D, Weinberg JS, Sawaya R, Lang FF, Ferson D, Heimberger AB, DeMonte F, Prabhu SS: Awake craniotomy for brain tumors near eloquent cortex: Correlation of intraoperative cortical mapping with neurological outcomes in 309 consecutive patients. Neurosurgery 64(5):836-346, 2009

21. Klimek M, Verbrugge SJC, Roubos S, van der Most E, Vincent AJ, Klein J: Awake craniotomy for glioblastoma in a 9-year-old child. Anaesthesia 59(6):607-609, 2004

22. Levin R, Hesselvik JF, Kourtopoulos H, Vavruch L: Local anesthesia prevents hypertension following application of the Mayfield skull-pin head holder. Acta Anaesthesiol Scand 33(4):277-279, 1989

23. Manninen $\mathrm{PH}$, Balki $\mathrm{M}$, Lukitto $\mathrm{K}$, Bernstein $\mathrm{M}$ : Patient satisfaction with awake craniotomy for tumor surgery: A comparison of remifentanil and fentanyl in conjunction with propofol. Anesth Analg 102(1):237-242, 2006

24. Moiyadi A, Shetty P: Early experience with combining awake craniotomy and intraoperative navigable ultrasound for resection of eloquent region gliomas. J Neurol Surg A Cent Eur Neurosurg 78(2):105-112, 2017

25. Moiyadi AV, Shetty PM, Mahajan A, Udare A, Sridhar E: Usefulness of three-dimensional navigable intraoperative ultrasound in resection of brain tumors with a special emphasis on malignant gliomas. Acta Neurochir (Wien) 155(12):22172225, 2013

26. Ozlu O: Anaesthesiologist's approach to awake craniotomy. Turk J Anaesthesiol Reanim 46(4):250-256, 2018

27. Pasquet $A$ : Combine regional and general anesthesia for craniotomy and cortical exploration. Part II. Anesthetic considerations. Anesth Analg 33:156-157, 1954

28. Penfield W, Pasquet A: Combined regional and general anesthesia for craniotomy and cortical exploration. Part 1. Neurosurgical considerations. Int Anesthiol Clin 24(3):1-20, 1986
29. Pereira LC, Oliveira KM, L'Abbate GL, Sugai R, Ferreira JA, da Motta LA: Outcome of fully awake craniotomy for lesions near the eloquent cortex: Analysis of a prospective surgical series of 79 supratentorial primary brain tumors with long follow-up. Acta Neurochir (Wien) 151(10):1215-1230, 2009

30. Piccioni F, Fanzo M: Management of anesthesia in awake craniotomy. Minerva Anestesiol 74(7-8):393-408, 2008

31. Potters JW, Klimek M: Local anesthetics for brain tumor resection: Current perspectives. Local Reg Anesth 11:1-8, 2018

32. Sacko O, Lauwers-Cances V, Brauge D, Sesay M, Brenner A, Roux FE: Awake craniotomy vs. surgery under general anesthesia for resection of supratentorial lesions. Neurosurgery 68(5):1192-1198; discussion 1198-1199, 2011

33. Sæther CA, Torsteinsen M, Torp SH, Sundstrøm S, Unsgård G, Solheim O: Did survival improve after the implementation of intraoperative neuronavigation and $3 \mathrm{D}$ ultrasound in glioblastoma surgery? A retrospective analysis of 192 primary operations. J Neurol Surg A Cent Eur Neurosurg 73(2):73-78, 2012

34. Sanus GZ, Yuksel O, Tunali Y, Ozkara C, Yeni N, Ozlen F, Tanriverdi T, Ozyurt E, Uzan M: Surgical and anesthesiological considerations of awake craniotomy: Cerrahpasa experience. Turk Neurosurg 25(2):210-217, 2015

35. Senel FC, Buchanan JM Jr, Senel AC, Obeid G: Evaluation of sedation failure in the outpatient oral and maxillofacial surgery clinic. J Oral Maxillofac Surg 65(4):645-650, 2007

36. Serletis D, Bernstein M: Prospective study of awake craniotomy used routinely and nonselectively for supratentorial tumors. J Neurosurg 107(1):1-6, 2007

37. Taylor MD, Bernstein M: Awake craniotomy with brain mapping as the routine surgical approach to treating patients with supratentorial intraaxial tumors: A prospective trial of 200 cases. J Neurosurg 90(1):35-41, 1999

38. Venkatraghavan L, Pasternak JJ, Crowley M: Anesthesia for awake craniotomy [UpToDate web site]. Available at: https://www.uptodate.com/contents/anesthesia-for-awakecraniotomy/print Accessed 20 11.2017.

39. Whittle IR, Midgley S, Georges H, Pringle AM, Taylor R: Patient perceptions of "awake" brain tumor surgery. Acta Neurochir (Wien) 147(3):275-277, 2005

40. Wilden JA, Voorhies J, Mosier KM, O'Neill DP, Cohen-Gadol AA: Strategies to maximize resection of complex, or high surgical risk, low-grade gliomas. Neurosurg Focus 34(2):E5, 2013

41. Yates JW, Chalmer B, McKegney FP: Evaluation of patients with advanced cancer using the Karnofsky performance status. Cancer 45(8):2220-2224, 1980 